\title{
Examining Choice in Self-directed Tiered Homework Assignments in College- Level Engineering Courses
}

\section{Dr. Vasiliki Ikonomidou, George Mason University}

Vasiliki N. Ikonomidou earned the Diploma in Engineering (MSc) degree and the PhD in Electrical Engineering from the Aristotle University of Thessaloniki, Greece, in 1997 and 2002 respectively. Her PhD thesis focused on the development of excitation techniques for magnetic resonance tagging. In May 2003 she joined the intramural program of the National Institute of Neurological Disorders and Stroke (NINDS) of the NIH (Bethesda, MD), as a Visiting Fellow in the Laboratory for Functional and Molecular Imaging, working in the development of high-contrast anatomical MRI techniques. In May 2006, she joined the Neuroimmunology Branch of NINDS, where she worked on brain imaging, using MRI and PET, in patients with Multiple Sclerosis. Since August 2009, she has been with George Mason University, where she is an Assistant Professor of Bioengineering. Her research interests are in the fields of video analytics for stress detection, MRI image processing, and differentiated learning techniques for engineering education. Dr Ikonomidou has co-authored 22 papers in peer-reviewed journals, and is a member of IEEE.

\section{Prof. Anastasia P Samaras, George Mason University, VA USA}

ANASTASIA P. SAMARAS is Professor of Education in the College of Education and Human Development at George Mason University, USA. She is an educational researcher and pedagogical scholar with signature work in self-study research methodology including co-editor of Polyvocal Professional Learning through Self-Study Research (2015) and author of Self-Study Teacher Research (2011) and lead editor of Learning Communities In Practice (2008). She is recipient of the Dissertation Research Award, University of Virginia, the Outstanding Scholar Award, University of Maryland, a Fulbright Scholar, and a Visiting Self-study Scholar. She served as chair of S-STEP from 2013-2015 and is a current Co-PI of two National Science Foundation (NSF) funded grants: Designing Teaching: Scaling up the SIMPLE Design Framework for Interactive Teaching Development and a research initiation grant: Student-directed differentiated learning in college-level engineering education. Her research centers on facilitating and studying her role in faculty development self-study collaboratives.

\section{Mr. Vikas Kotari, George Mason University}

Vikas Kotari is a PhD candidate in the electrical and computer engineering department at George Mason University, Fairfax, VA. He earned his M.S in electrical engineering from George Mason University in Dec 2009. His $\mathrm{PhD}$ thesis focuses on characterization and longitudinal tracking of multiple sclerosis (MS) lesions in brain magnetic resonance images. Vikas uses image registration, subtraction imaging, and segmentation algorithms among other image processing and image analysis techniques to characterize and track MS lesions changes. Vikas has interned at Genentech, South San Francisco. During this time he has developed an image analysis algorithm to segment in-vivo volumetric micro-computed tomography mice data. In addition, Vikas has developed a multispectral fusion based detection technique for Virchow-Robin spaces. His research interests include medical image processing, texture analysis for evaluating accuracy of registration, linear and non-linear image registration techniques and, image segmentation algorithms for segmenting brain magnetic resonance data. 


\section{Examining Choice in Self-directed Tiered Homework Assignments in College-Level Engineering Courses}

\section{Introduction}

The term "differentiated instruction" 1,2 has been used in the context of K-12 education in order to describe a set of strategies aiming at providing individualized instruction for students at different readiness levels. It describes an instructional design in which, while the class as a whole still follows a common instruction plan, the incorporation of activities that students perform, either individually or in groups, allows for an additional component that the student and the teacher have the opportunity to adjust to the ability level of individual students.

Within the differentiated instruction paradigm, students with lower readiness levels may spend time developing simpler basic skills that are needed before they can advance to the more challenging activities while students exhibiting higher readiness levels may be exposed to more challenging activities that will be helpful to further develop their potential. Current literature suggests that differentiated instruction is an effective option for designing instruction in classrooms where there is significant difference in students' academic readiness levels. ${ }^{3-5}$

While conceptually simple, the implementation of this plan in K-12 is not without challenges. ${ }^{6,7}$ Students need to ultimately meet the same educational objectives. This means that the instructor needs to carefully design different instructional pathways to assure that the target levels of material mastery are reached by all students. What this means is that a plan is needed where students at higher readiness levels focus on higher levels of mastery, such as analysis or design, while students with lower readiness levels are afforded scaffolded opportunities to learn the material and not spend their time memorizing facts. This leads to the problem of designing and executing a multitude of different instructional activities that ultimately lead to the same educational objectives, albeit at possibly different levels, for all students in the class. Also necessary in the design are appropriate assessments and pathways for students with differentiated readiness levels.

Despite the success of differentiated instruction techniques at the K-12 level, there is very limited literature for the application of such an approach to college-level education ${ }^{8,9}$, with most of it being focused to remedial courses, where students may be offered a possibility of "testing out" of different parts of the course. This may represent a missed opportunity, since evidence suggests that different readiness levels are a reality in college classrooms, and with it come different student needs in order to succeed. One possible reason for this is the commitment on behalf of the instructor that is necessary in the K-12 model of differentiated learning: in it, the teacher is tasked with creating a variety of mini-curricula suited to the students' individual needs. Such a level of involvement may be not realistic for engineering college faculty.

To overcome this, we turned to a well-known concept from adult learning theory, namely self-directed learning, a process in which learners take the initiative to identify their needs, formulate goals, identify resources, choose and implement learning strategies and assess learning outcomes ${ }^{10}$. As Knowles points out in ${ }^{10}$, it is a process that takes place in collaboration with 
others, such as teachers and peers; and it is considered an important step for students in their transition to life-long learning. Given that engineering students are adults, the larger question of this study was whether we could develop interventions that allow engineering students to implement differentiated learning adjusted to their own needs, while shifting the choice to them rather than to the faculty. In order to achieve this, we introduced a set of interventions, consisting of detailed objective rubrics, formative assessments and tiered homework assignments, and with problem "menus" at different challenge levels that students could choose from. In this paper, our interest is in the factors that influence student choice when faced with tiered assignments within the context of a college-level engineering course. For this, we analyze data collected from a student cohort over the period of a semester. Our primary hypothesis was that student choice of assignment challenge levels would be correlated with the students' readiness levels for the class, leading to a student selection mechanism similar to the active intervention of the teacher in the K-12 model.

\section{Methods}

\section{Context}

The course selected as the testbed for this study is a sophomore-level class in a Bioengineering curriculum at a large public University, and is one of the first engineering courses students encounter in this program. Data for this study were collected during the Spring 2015 semester. There were 44 students who provided written informed consent under an IRB approved protocol to have their course data analyzed for this study. From that group, 40 students completed the class and 4 dropped the class for reasons unrelated to the study.

\section{Pedagogical Interventions}

Class material is structured in four units. The first intervention, aimed at supporting the students in both identifying needs and formulating goals was the introduction of detailed rubrics stating the learning aims and skills for each unit. These rubrics went into more detail than the learning objectives stated in the course syllabus, and were designed to serve as a study guide for the students. The second intervention was a first short homework assignment for each unit, which was graded for effort only. The purpose of this assignment was to give students formative assessment, by allowing them to test their skills in a safe environment, as the impact of any mistakes on that homework assignment would be minimal.

Our key pedagogical intervention tested in the study was a tiered homework assignment. In our design, these homework assignments consisted of problem menus organized in three categories, corresponding to the levels of apply, analyze and creating (termed "design") in Bloom's taxonomy. Each problem was assigned a number of points, and students were asked to choose problems in order to earn a certain number of points. A student could not complete the number of points required for passing by solving problems in the "apply" or "analyze" sections alone. The challenge was to encourage and support students' extended reach towards the "creating" or design level. Four such assignments were presented over the course of a semester; each such homework was preceded by a conventional assignment that was graded for effort only, aiming at providing formative feedback to the student prior to the tiered assignment. Additionally, each tiered assignment was accompanied by a brief, open-ended questionnaire aiming at understanding how students chose problems to solve in this context. Questions included were: 
- Why did you choose the problems that you solved?

- How do you think the level of this assignment compares to the level expected of the class, as specified in the rubrics provided? Why?

- Do you think the level of difficulty of the assignments is reflected correctly in the points assigned to each problem?

\section{Results}

Our main interest in this design was in the first question: "Why did you choose the problems that you solved?" Here, confidence that they could solve the problem was the strongest factor, with answers ranging from $80 \%$ to $86 \%$ over the four assignments. References to confidence as a reason for choice drop slightly over the course of the semester.

Learning, which can be expressed either as building better understanding or as preparing for the exam was the second most cited reason, with answers ranging from $17 \%$ to $25 \%$. Interestingly though, it is only in the last assignment that we see answers like "I chose these problems because these problems were ones that I needed more focus on. It helped me understand the processes and calculations" or "I choose the easier problems because I was very uncertain of how to solve these types of questions. I'm glad I did, because I felt very confident after solving them", indicating that understanding the purpose of the tiered homework takes several cycles.

Time constraints, usually coupled with a rejection of the more complicated design questions, ranged from $6 \%$ to $17 \%$ with concerns increasing as the semester progressed. This correlated with a rejection of the more time-consuming design problems, with students claiming that they did not have the time to solve them, and opting for the less time-consuming apply or analyze questions instead.

At the same time, looking for a challenge, which we initially assumed would motivate students to choose design problems, started at $16 \%$ and dropped to $10 \%$ towards the end of the semester. In looking further into the results, we noticed three interesting trends: (a) There was a trend of challenge rejection due to the risk of losing possible points, as noted in this characteristic answer: "I considered trying some of the design problems, but I did not want to risk losing points for inaccurate work". Similarly comments refer to the possibility of grading design questions for effort only, so that students would feel comfortable taking a chance with them. (b) Contrary to our original thought that "challenge" would represent a choice of the more difficult design problems, students noted as challenge going from the more straightforward apply problems into the analyze ones, as stated in this answer: "I chose the above problems because I wanted to make sure I understood the basics in the application part of the homework. It is very important to understand the basics and the reason behind doing everything before actually solving some problems. Then I wanted to do a challenging analysis question that required me to create the equations and solve for them". (c) While our original hypothesis was that students with higher readiness levels would be motivated by challenge and select the more involved design questions, this proved to be false, as the majority of the students who consistently performed well in the first, formative homework assignments seemed to prefer a more conservative approach that would maximize their grades instead. There was a small number of high-performing students that 
showed a strong preference for the design problems, however our sample size is too limited to extract any conclusions.

Answers to the second question "How do you think the level of this assignment compares to the level expected of the class, as specified in the rubrics provided? Why?" reveal an interesting trend: At the beginning of the semester, students perceive the tiered homework assignment as very challenging and time-consuming, and a main cause for the overall challenge seems to be the need to deviate from class material in both the analyze and the design questions. Answers become more positive towards the end of the semester, where students still perceive the assignments as mildly difficult and challenging, but still time-consuming. A few students mentioned that they did not use the rubrics at all, however most of them claimed that when compared to the rubrics, the level of the homework corresponded well to what was described in the rubrics.

The third question, "Do you think the level of difficulty of the assignments is reflected correctly in the points assigned to each problem?" asks students to give their opinion on the points attributed to each problem. Here, students will usually agree that the more difficult problems give the more points; however, there are some complains about the points assigned to apply level problems as some students do seem to think that the reward mechanism should rather be based on time spent on an assignment.

\section{Discussion}

Differentiated instruction techniques have provided a successful solution in K-12 for addressing challenges related to varied student readiness levels in the classroom. Its working hypothesis is that by adjusting the curriculum requirements to the needs of the individual student, it is possible to better support student performance and growth. In this study, we tried to adapt the concept of differentiated instruction to a college-level engineering course, with one major change from the differentiated learning paradigm usually presented in literature: in our intervention, it was the student choosing between assignments of different levels of difficulty instead of the instructor This analysis aims at understanding how students make choices in such a setting.

As indicated by responses to the third question "Do you think the level of difficulty of the assignments is reflected correctly in the points assigned to each problem?" as well as further comments within the open-ended responses, students agree with the instructor's perception of difficulty of the problems. In general, "apply" questions are perceived as easier than "analyze", and "design"-level questions are thought as the most challenging ones. However, time required to complete an assignment might interfere with this perception: conceptually simpler time-consuming problems may occasionally be perceived as more challenging, indicating that devoting time to the homework assignment presents a challenge of its own, independent of the intellectual challenge presented by the assignment problems.

Two tools were offered to the students to support them in assessing their own readiness levels: a set of rubrics detailing the course requirements for each unit, and a first homework assignment in each unit graded for effort only, designed to act as a formative assessment tool. Interestingly, neither the rubrics nor performance in the first formative assignment were mentioned directly as 
factors in the choice of problems in the tiered homework. At this stage, it is not clear whether this is because rubrics and the first homework did not play a role in this selection, or because students did not think to mention them in their short open answers. Based on further data collection, rubrics become important for the students as study guides for exams, but their support in homework selection is unclear. As this is an ongoing project, two steps are planned to help better assess this point: (a) introduce a self-grading step for the first homework assignment, to avoid students ignoring its results based on a good grade given for effort only, and (b) refine our questions to probe specifically for the rubric and the effect of the first homework assignment.

The main trend that emerged from our results is that confidence in their ability to solve the assignment problems was the main factor for student choice. Given that most students perceived the homework as "difficult" or "challenging", as indicated by answers to the question "How do you think the level of this assignment compares to the level expected of the class, as specified in the rubrics provided," this indicates that students still identify a comfort zone within the options offered, and choose accordingly. Cost, in terms of time spent and possible loss of points if they unsuccessfully attempt to solve a more challenging problem seem to reinforce the need for staying within a comfort zone, and this is a pattern that persists even among students with higher readiness levels.

Seeking intellectual challenge was less pronounced than originally expected. There are three main patterns that seem to emerge with respect to that. First, the analyze options, where students were expected to handle a more situational verbal description of a problem and evaluate their results seemed to be more challenging than expected, as many students noted that this was different from experiences in their previous courses. Given that this is one of the first engineering courses these students take, it would be interesting to compare this with results in a higher-level engineering course. Secondly, design choices were both high risk in terms of possible point loss, and more time-consuming, making them less desirable for students whose main goal was to get a good grade in the class. To mitigate this, introducing a different grading policy for design problems may be considered. Lastly, it was noted that there were a few students that consistently claimed that they enjoyed the challenge, and who chose the design problems in the assignment; however, not all of these students were the ones with the highest readiness levels, pointing more to a character trait than a high confidence level.

Other than complaints about the time requirements for completing the assignment, students perceived the interventions as useful. This was reflected in the course ratings, which improved by $15 \%$ with respect to previous semesters. Overall student performance seemed to benefit as well: Students scored an average of $67 \%$ in the midterm exam, but a $77 \%$ in the final exam, with the most notable improvements in the lowest-performing students. In comparison, average scores in the previous year averaged to $63 \%$ in the midterm exam and $68 \%$ in the final exam. However, these results need to be interpreted taking into account the lack of a true control group; we are considering the possibility of a future class offering featuring a cross-over design, where half the units will have conventional assignments, and half will have tiered assignments, which will allow to compare student performance between the two designs.

From the instructor's standpoint, development of the intervention required significant effort, but 
it can serve as a starting point for applying the intervention in subsequent semesters. However, grading a tiered homework exam proved more complicated and more time intensive than grading a conventional homework assignment, especially if the aim is to give sufficient individualized feedback to the student. For this, the development of detailed assignment grading rubrics is the next step.

Based on both feedback received and student performance, the introduction of tiered homework assignments in a sophomore level Bioengineering course is deemed a successful intervention. Data collection is currently ongoing in order to better understand how an instructor might further explore/ design an environment that encourages students to take challenges while at the same time supporting students at lower readiness, or even confidence levels. Further planned interventions include strengthening the role of the formative assessments by introducing a self-grading step, and the development of a video library of solved example questions to provide additional support for students in attempting problems outside of their level of comfort.

\section{References}

1. Tomlinson, C. A. How to differentiate instruction in mixed-ability classrooms. (Association for Supervision and Curriculum Development, 2001).

2. Tomlinson, C. A. The Differentiated Classroom: Responding to the Needs of All Learners. (2001).

3. Levy, H. M. Meeting the Needs of All Students through Differentiated Instruction: Helping Every Child Reach and Exceed Standards. Clear. House J. Educ. Strateg. Issues Ideas 81, 161-164 (2008).

4. Cusumano, C. \& Mueller, J. How Differentiated Instruction Helps Struggling Students. Leadership 36, 8-10 (2007).

5. McTighe, J. \& Brown, J. L. Differentiated instruction and educational standards: Is détente possible? Theory Pract. 44, 234-244 (2005).

6. Tomlinson, C. A. et al. Differentiating Instruction in Response to Student Readiness, Interest, and Learning Profile in Academically Diverse Classrooms: A Review of Literature. J. Educ. Gift. 27, 119-145 (2003).

7. Tomlinson, C. A. \& Strickland, C. A. Differentiation in practice: a resource guide for differentiating curriculum, grades 9-12. (Association for Supervision and Curriculum Development, 2005).

8. Chamberlin, M. \& Powers, R. The promise of differentiated instruction for enhancing the mathematical understandings of college students. Teach. Math. Its Appl. 29, 113-139 (2010).

9. Santangelo, T. \& Tomlinson, C. A. The Application of Differentiated Instruction in Postsecondary Environments: Benefits, Challenges, and Future Directions. Int. J. Teach. Learn. High. Educ. 20, 307-323 (2008).

10. Knowles, M. S. Self-Directed Learning: A Guide for Learners and Teachers. (1975). 